\title{
Factores de riesgo de alteraciones citológicas del cuello uterino en mujeres chilenas: Un estudio de casos y controles
}

\author{
MARÍA TERESA SOLÍS ${ }^{1}$, FRANCISCO AGUAYO ${ }^{2 a}$, \\ MACARENA VARGAS ${ }^{2 \mathrm{~b},}$ FABIOLA OLCAY ${ }^{3 \mathrm{c}}$, KLAUS PUSCHEL ${ }^{4}$, \\ ALEJANDRO CORVALÁN ${ }^{2}$, CATTERINA FERRECCIO ${ }^{1}$
}

\section{Risk factors associated with abnormal cervical cytology among Chilean women: A case control study}

Background: Cervical cancer is the third cause of cancer death among Chilean women, affecting mainly women from low socioeconomic status. Aim: To determine main risk factors $(R F)$ including human papilomavirus (HPV) types associated with abnormal cervical cytology (Atypical Squamous Cells of Undetermined Significance or ASCUS) among Chilean women from low socioeconomic status in Santiago, Chile. Material and Methods: A random population based sample of 616 women from La Pintana (a low-income district in Santiago) participated in 2001 in a HPV prevalence study and were re-evaluated in 2006 through a risk factors questionnaire, Papanicolaou test and DNA detection for HPV. The Papanicolaou test was analyzed in Santiago and HPV analysis (PCR_GP5+/GP6+) was conducted in Vrije University, Amsterdam. Cases included 42 women with cervical lesions and controls included 574 women with normal cytology during the period 2001-2006. Logistic regression with uni and multivariate analysis was performed to identify RF for cervical lesions. Results: During the study period, there was a significant increase in the proportion of single women, from 8.3 to $14.8 \%$ ( $p<0.05)$, of women with 3 or more sexual partners from 8.9 to 13.3 and of women high risk HPV, from 9.1 to $14.3 \%$. The proportion of abnormal Papanicolaou tests remained stable (3.08 and 3.9\% > ASCUS). High risk HPV was the most significant factor associated with cervical lesions (odds ratio $(O R)=9.695 \%$ confidence intervals $(C I)=4.4-21.1)$ followed by oral contraceptive use $(O R=2.5895 \% C I=1.2-5.7)$. Among women infected by high risk $H P V$, the use of oral contraceptives was a risk factor while compliance with screening was protective for cervical lesions. Conclusions: From 2001 to 2006, there was an increase in the proportion of women with high risk HPV infections.

(Rev Med Chile 2010; 138: 175-180).

Key words: Cytology; Human papilloma virus; Risk factors.

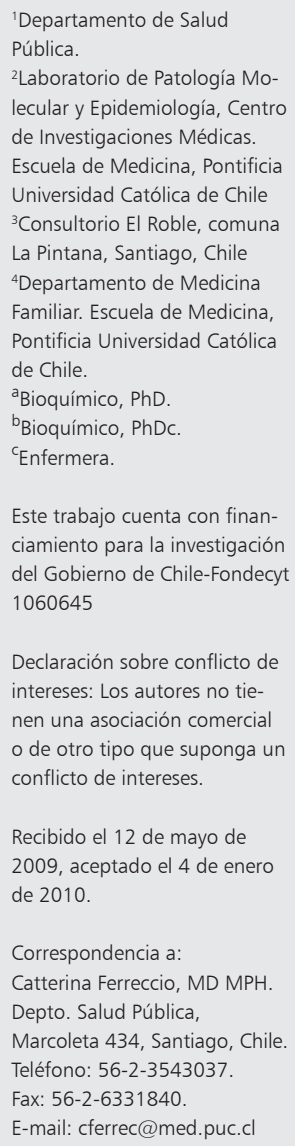

1 1 cáncer cervicouterino es el segundo cáncer más común en mujeres a nivel mundial con 274.000 muertes el año 2002, presentándose $83 \%$ de los casos en los países en vías de desarrollo ${ }^{1}$. En Chile, el programa de prevención de cáncer cervicouterino basado en el examen del
Papanicolaou cada tres años en mujeres entre 25 y 64 años $^{2}$, se ha asociado con una disminución de la tasa de mortalidad por este cáncer. El año 2005 el cáncer cervicouterino alcanzó una tasa de 8,2 por 100.000 mujeres, pasando del segundo al tercer lugar de la mortalidad por cáncer en las 
mujeres, precedido por el cáncer de mama y de vesícula biliar ${ }^{3}$. A pesar del avance en el promedio nacional, existe una diferencia importante de riesgo entre mujeres de nivel socioeconómico alto y bajo. Entre 1985 y 1995 la mortalidad por cáncer cervicouterino bajó 51,6\% y $12,5 \%$ en las mujeres con 12 años o más de educación versus aquellas con menos de 8 años, respectivamente ${ }^{4}$. La Agencia Internacional de Investigación de cáncer (IARC) en 1995 declaró al virus papiloma humano (VPH) como causa necesaria para cáncer cervicouterino ${ }^{5,6}$; pero se reconoce que la sola infección por VPH no es una causa suficiente puesto que $90 \%$ a 95\% de las mujeres eliminan el virus sin desarrollar cáncer cervicouterino ${ }^{7}$. Se han distinguido factores de riesgo de adquirir una infección por VPH y factores de riesgo de desarrollar un cáncer cervicouterino, dado que la mujer se ha infectado. Entre los primeros se cuentan edad precoz de la primera relación sexual y mayor número de parejas sexuales en la vida ${ }^{7,8}$. Entre los segundos se ha descrito un alto número de partos ${ }^{9}$, uso de anticonceptivos orales $(\mathrm{ACO})^{10} \mathrm{y}$ tabaquismo ${ }^{11,12}$. En Chile no se ha investigado los factores asociados a presentar lesiones cervicouterinas, siendo particularmente relevante entre los subgrupos poblacionales en que persisten tasas de mortalidad evitables. El presente estudio tuvo como objetivos medir los factores de riesgo de infección por VPH y de lesiones cervicouterinas en mujeres chilenas mayores de 17 años residentes en un área de bajos recursos económicos de Santiago. El protocolo del estudio fue aprobado por el comité de ética Escuela de Medicina de la Pontificia Universidad Católica de Chile y por el Servicio de Salud Sur-Oriente.

\section{Material y Métodos}

\section{Población de origen}

El grupo de estudio es una cohorte de $616 \mathrm{mu}$ jeres $>$ de 17 años, que se ingresaron el año 2001 a un estudio de prevalencia de VPH en Santiago. La metodología del estudio de base ya ha sido descrita previamente ${ }^{13}$. En breve, se trata de una muestra aleatoria de la población residente en el área de cobertura del Centro de Salud El Roble en la comuna de La Pintana. Las mujeres fueron contactadas en su domicilio, donde se les aplicó una encuesta sobre factores de riesgo y luego se invitó al Centro de Salud donde se les realizó un examen ginecológico y se les tomó muestras para estudio de Papanicolaou y de VPH, a todas aquellas que presentaron alteraciones se las citó a control de acuerdo a las normas vigentes en ese momento. Todas las mujeres fueron reexaminadas, con igual metodología, 5 años más tarde, en 2006. Se siguió retrospectivamente los resultados de los Papanicolaou y de consultas ginecológicas que las mujeres se realizaron en el intervalo 2001-2006.

\section{Casos de estudio}

Se eligió como punto de corte de citología anormal un Papanicolaou con lesión atípica de significado indeterminado (ASCUS), criterio que permite maximizar la sensibilidad de este méto$\mathrm{do}^{14}$. Se definieron como casos, las mujeres que presentaron un diagnóstico citológico de ASCUS o peor, detectado durante el año 2001 o 2006 (n $=42$ ). Los controles fueron las mujeres con diagnóstico citológico normal en todo el período del estudio $(\mathrm{n}=574)$.

\section{Técnicas de laboratorio}

Las muestras de Papanicolaou fueron teñidas y leídas por citopatólogos entrenados, bajo la supervisión de un médico patólogo. El ADN de VPH fue analizado en el laboratorio de patología de Vrije University, Amsterdam, Holanda, a través de reacción de polimerasa en cadena (PCR), usando los partidores GP5+/GP6+ para amplificar un fragmento del gen L1 de VPH (155 bp $)^{15}$. Los fragmentos obtenidos a través de la amplificación mediante PCR con los partidores GP5+/6+ se analizaron con un ensayo inmuno-enzimático (EIA) para determinar la presencia de un amplio espectro de genotipos de VPH usando dos cócteles de sondas específicas ${ }^{16}$. Un cóctel contenía sondas para genotipos de alto riesgo $(16,18,26,31,33$, $35,39,45,51,52,53,56,58,59,66,68,67,73$ у $82)$ y otro cóctel contenía sondas para genotipos de riesgo bajo e indeterminado $(6,11,34,40$, $42,43,44,54,61,70,71,72,81$ y CP6108). Para genotipificar, los productos de PCR positivos en PCR/EIA, se usó reverse line blotting (RLB), con métodos previamente descritos ${ }^{16}$. Se consideró a una mujer positiva para infección por VPH de alto riesgo, cuando se identificó cualquiera de los tipos de alto riesgo epidemiológico descritos por Muñoz et al. ${ }^{17}$.

Estadística: El riesgo de presentar alteración citológica cervicouterina, se modeló con regresión 
logística múltiple, calculándose odds ratios (ORs) e intervalos de confianza 95\% (95\% CI). Se calcularon los OR para todas las mujeres y aparte para aquellas infectadas con VPH. La información fue analizada utilizando el software estadístico SPSS versión 15.

\section{Resultados}

En 616 mujeres se tuvo información completa el año 2001 y el 2006 (Tabla 1), al comparar el estudio basal con el re-examen el 2006, las mujeres son
5 años mayores, aumentaron las que se declaran solteras y las que reportan más de tres parejas en la vida. Aunque el total de alteraciones citológicas no cambió significativamente, aumentó el diagnóstico de ASCUS en desmedro de las lesiones de bajo o alto grado. Tuvieron un aumento significativo en la frecuencia de infección por VPH (Tabla 1). En los cinco años de seguimiento, 42 mujeres fueron clasificadas como casos porque presentaron un Papanicolaou calificado como ASCUS o peor, detectadas al inicio en el año 2001 (19 mujeres), en el seguimiento el 2006 (22 mujeres), o en ambos

Tabla 1. Características de las 616 mujeres estudiadas y cambios en el período de estudio. La Pintana 2001-2006

\begin{tabular}{|c|c|c|c|c|}
\hline & & $\begin{array}{c}2001 \\
(n=616) \\
\%\end{array}$ & $\begin{array}{c}2006 \\
(n=616) \\
\%\end{array}$ & $\begin{array}{c}\text { valor } \mathbf{p} \\
\text { del cambio* }\end{array}$ \\
\hline Edad & $\leq 30$ años & 18,8 & 11,9 & $<0,05$ \\
\hline \multirow[t]{3}{*}{ Situación conyugal } & Viuda & 14 & 17 & 0,16 \\
\hline & Casada & 77,8 & 68,2 & $<0,05$ \\
\hline & Soltera & 8,3 & 14,8 & $<0,05$ \\
\hline Años de estudio & $\leq 8$ & 62,0 & 62,4 & 0,90 \\
\hline Edad primera relación sexual (años) & $\leq 17$ & 43,7 & 42,1 & 0,60 \\
\hline Paridad & $>2$ partos & 60,4 & 62,9 & 0,39 \\
\hline Antecedentes ITS & & 4,2 & 4,7 & 0,77 \\
\hline \multirow[t]{3}{*}{ Número de parejas sexuales } & 1 & 63,5 & 61,8 & 0,58 \\
\hline & 2 & 27,6 & 24,9 & 0,31 \\
\hline & $\geq 3$ & 8,9 & 13,3 & $<0,05$ \\
\hline Usó ACO (alguna vez) & & 39,4 & 35,9 & 0,22 \\
\hline Usó Preservativo & & 13,5 & 21,4 & $<0,05$ \\
\hline Pap (alguna vez) & & 82,8 & 98,9 & $<0,05$ \\
\hline Tabaquismo actual & & 37,2 & 31,5 & $<0,05$ \\
\hline \multirow[t]{4}{*}{ Pap alterado } & ASCUS & 1,5 & 3,2 & $<0,05$ \\
\hline & Lesiones de bajo grado & 0,7 & 0,5 & $1,00^{¥}$ \\
\hline & Lesiones de alto grado & 1 & 0,2 & $0,07^{¥}$ \\
\hline & Total lesiones ( $\geq$ ASCUS) & 3,08 & 3,9 & 0,53 \\
\hline \multirow[t]{4}{*}{ Infección VPH } & VPH Alto riesgo & 9,1 & 14,3 & $<0,05$ \\
\hline & VPH Bajo riesgo** & 2,8 & 7,3 & $<0,05$ \\
\hline & VPH Riesgo Indeterminado*** & 0,8 & 1,5 & 0,40 \\
\hline & Total VPH & 12,58 & 23,17 & $<0,05$ \\
\hline
\end{tabular}

*comparación frecuencias en el año 2006 versus 2001. **Excluye mujeres con VPH de alto riesgo. ***Incluye los genotipos de VPH 67, 70, 73. ${ }^{*}$ Prueba exacta de Fisher. 
Tabla 2. Factores de riesgo de Citología cervical anormal en 616 mujeres. La Pintana 2001-2006

\begin{tabular}{|c|c|c|c|c|c|c|}
\hline $\begin{array}{l}\text { Características } \\
\text { ( Año 2000) }\end{array}$ & $\begin{array}{c}\text { Casos* } \\
(42) \\
\%\end{array}$ & $\begin{array}{c}\text { Controles } \\
\text { (574) } \\
\%\end{array}$ & $\begin{array}{c}\text { OR } \\
\text { Crudo }\end{array}$ & IC (95\%) & $\begin{array}{c}\text { OR }^{* *} \\
\text { Ajustado }\end{array}$ & IC (95\%) \\
\hline VPH Alto riesgo & 44,1 & 7,0 & 10,42 & $(4,9-22,0)$ & 9,60 & $(4,4-21,1)$ \\
\hline VPH Bajo riesgo (solo) & 2,9 & 2,8 & 1,05 & $(0,1-8,1)$ & - & - \\
\hline Edad $\leq 30$ años & 26,2 & 18,3 & 1,59 & $(0,8-3,3)$ & 0,50 & $(0,1-1,7)$ \\
\hline Uso ACO & 50,0 & 38,7 & 1,59 & $(0,8-3,0)$ & 2,58 & $(1,2-5,7)$ \\
\hline Tabaquismo actual & 45,2 & 36,6 & 1,43 & $(0,8-2,7)$ & 1,36 & $(0,6-2,9)$ \\
\hline Papanicolaou previo & 71,4 & 83,6 & 0,49 & $(0,2-1,0)$ & 0,40 & $(0,1-1,3)$ \\
\hline$\leq 8$ años de estudio & 47,6 & 62,9 & 0,54 & $(0,3-1,0)$ & - & - \\
\hline$>2$ partos & 54,8 & 60,8 & 0,78 & $(0,4-1,5)$ & - & - \\
\hline \multicolumn{7}{|l|}{ Situación conyugal } \\
\hline Separada/Viuda & 14,3 & 13,9 & 1 & - & - & - \\
\hline Casada/concubina & 78,6 & 77,7 & 0,83 & $(0,2-3,5)$ & - & - \\
\hline Soltera & 7,1 & 8,4 & 0,99 & $(0,4-2,4)$ & - & - \\
\hline Primera relación sexual $\leq 17$ años & 46,3 & 43,6 & 1,12 & $(0,6-2,1)$ & - & - \\
\hline \multicolumn{7}{|l|}{ Número parejas sexuales (vida) } \\
\hline 1 pareja & 52,4 & 64,3 & 1 & - & 1 & - \\
\hline 2 parejas & 40,5 & 26,7 & 1,86 & $(1,0-3,6)$ & 1,44 & $(0,6-3,2)$ \\
\hline$\geq 3$ parejas & 7,1 & 9,1 & 0,97 & $(0,3-3,3)$ & 0,77 & $(0,2-3,1)$ \\
\hline Uso preservativo & 19,0 & 13,1 & 1,57 & $(0,7-3,5)$ & - & - \\
\hline Antecedente ITS & 2,4 & 4,4 & 0,54 & $(0,1-4,1)$ & - & - \\
\hline
\end{tabular}

$\mathrm{VPH}=$ virus papiloma humano, $\mathrm{ACO}=$ anticonceptivos orales, en negrilla las asociaciones estadísticamente significativas (valor p < 0,05); *Papanicolaou anormal año 2001-2006; **Análisis ajustado por las siguientes variables: VPH Alto Riesgo, edad, uso ACO, tabaquismo, Pap previo, número de parejas sexuales. Bondad de ajuste del modelo multivariado: Prueba de Hosmer y Lemeshow = 0,64 (Chi cuadrado 6,09) Curva ROC: Área= 0,7 7 IC 95\% (0,68-0,85); Error típico= 0,04; Valor $p=<0,05$.

momentos (1 mujer); no se detectó casos en el intervalo 2001-2006. Como controles calificaron 574 mujeres que tuvieron un diagnóstico citológico normal en los exámenes del año 2001 y del 2006 y que no tuvieron Papanicolaou alterado en el intervalo.

Resultaron factores de riesgo de ASCUS la infección por VPH de alto riesgo (AR) y haber tenido más de dos parejas, estando en el límite de la significación la edad menor de 30 años, uso de anticonceptivos orales (ACO), tabaquismo actual. Sin embargo, al ajustar estas variables en un modelo multivariado sólo persisten con significación estadística la infección por VPH de AR y el uso de ACO (Tabla 2). Por otro lado, el único factor de riesgo de alteración citológica cervicouterina entre las 55 mujeres infectadas por VPHAR fue el uso de ACO, en tanto resultó un factor protector haberse realizado al menos un Papanicolaou en la vida (Tabla 3 ).

\section{Discusión}

Este es el primer estudio chileno que analiza factores de riesgo asociados a Papanicolaou alterado en la población general, siendo lo más destacable el importante rol preventivo del tamizaje entre las mujeres ya infectadas por el VPH. Es interesante notar que en este grupo de mujeres, en un intervalo de sólo 5 años aumentó significativamente la infección por VPH, sin acompañarse 
Tabla 3. Factores de riesgo asociados a citología alterada en 55 mujeres infectadas por VPH de alto riesgo oncogénico. La Pintana 2001-2006

\begin{tabular}{|c|c|c|c|c|c|c|}
\hline & $\begin{array}{l}\text { Casos* } \\
\text { (15) \% }\end{array}$ & $\begin{array}{l}\text { Controles** } \\
\text { (40) } \%\end{array}$ & $\begin{array}{c}\text { OR } \\
\text { Crudo }\end{array}$ & $\begin{array}{c}\text { IC } \\
(95 \%)\end{array}$ & $\begin{array}{l}\text { OR *** } \\
\text { ajustado }\end{array}$ & $\begin{array}{c}\text { IC } \\
(95 \%)\end{array}$ \\
\hline Papanicolaou previo & 66,7 & 90,0 & 0,22 & $(0,1-1,0)$ & 0,11 & $(0,0-0,8)$ \\
\hline Edad $\leq 30$ años & 26,7 & 15,0 & 2,06 & $(0,5-8,7)$ & 0,27 & $(0,0-2,6)$ \\
\hline Uso ACO & 66,7 & 42,5 & 2,71 & $(0,8-9,4)$ & 5,68 & $(1,0-32,5)$ \\
\hline \multicolumn{7}{|l|}{ Situación conyugal } \\
\hline Separada/Viuda & 13,3 & 22,5 & 1 & - & - & - \\
\hline Casada & 80,0 & 67,5 & 2,00 & $(0,4-10,7)$ & - & - \\
\hline Soltera & 6,7 & 10,0 & 1,13 & $(0,1-16,3)$ & - & - \\
\hline$\leq 8$ años de estudio & 53,3 & 70,0 & 0,49 & $(0,1-1,7)$ & - & - \\
\hline Primera relación sexual $\leq 17$ años & 53,3 & 55,0 & 0,94 & $(0,3-3,1)$ & - & - \\
\hline \multicolumn{7}{|l|}{ Número parejas sexuales } \\
\hline 1 pareja & 33,3 & 45,0 & 1 & - & 1 & - \\
\hline 2 parejas & 53,3 & 40,0 & 1,80 & $(0,5-6,6)$ & 2,73 & $(0,5-13,6)$ \\
\hline$\geq 3$ parejas & 13,3 & 15,0 & 1,20 & $(0,2-7,9)$ & 1,31 & $(0,2-11,1)$ \\
\hline Uso preservativo & 6,7 & 7,5 & 0,88 & $(0,1-9,2)$ & - & \\
\hline Tabaquismo actual & 53,3 & 40,0 & 1,71 & $(0,5-5,7)$ & 0,71 & $(0,2-3,2)$ \\
\hline Paridad $>2$ & 53,3 & 70,0 & 0,49 & $(0,1-1,7)$ & - & - \\
\hline
\end{tabular}

$\mathrm{VPH}=$ virus papiloma humano, $\mathrm{ACO}=$ anticonceptivos orales, en negrilla las asociaciones estadísticamente significativas (valor $\mathrm{p}<0,05)$; ${ }^{*}$ Casos VPH positivos y Pap alterado. ${ }^{* *}$ Controles, VPH + y Pap normal. ***Análisis ajustado por las siguientes variables: Pap previo, edad, ACO, No parejas, Tabaquismo, Bondad de ajuste del modelo multivariado: Prueba de Hosmer y Lemeshow = 0,97 (Chi cuadrado 1,42 ) Curva ROC: Área =0,7 5 IC 95\% (0,60-0,90); Error típico =0,08; Valor p $=<0,05$.

aún de aumento de las alteraciones citológicas. Este incremento de la infección por VPH, se puede explicar por una mayor exposición a nuevas parejas sexuales, en efecto, en el período aumentaron las mujeres que se declaran solteras y las que declaran más de tres parejas sexuales en la vida. Los cambios observados en esta cohorte son consistentes con otros estudios nacionales recientes que reportan tendencia a adelantar la edad de inicio de actividad sexual y un aumento en el número de compañeros sexuales en la vida ${ }^{18}$.

En nuestra serie, sólo los ACO fueron cofactores significativos del VPH para causar alteraciones citológicas. Se ha sugerido que los ACO provocarían un aumento en la incidencia de ectropión cervico uterino, favoreciendo una mayor exposición de la unión escamo-columnar a potenciales agentes carcinogénicos. Se ha reportado que el estrógeno y la progesterona también podrían afectar directamente las células cervicouterinas, aumentando la proliferación celular y estimulando la actividad transcripcional (transactivación) de los oncogenes E6 y E7 de VPH ${ }^{19}$.

Por otro lado, el uso del Papanicolaou fue el único factor protector. El efecto protector del Papanicolaou es ampliamente reconocido en los países desarrollados y también se ha asociado a un descenso de mortalidad por cáncer cervicouterino en algunos países latinoamericanos ${ }^{20-21}$.

En conclusión, el grupo en estudio presentó aumento de riesgo de infección por VPH, siendo el uso del tamizaje una estrategia efectiva para prevenir lesiones. Será necesario reforzar el tamizaje para evitar que el aumento de las infecciones por VPH AR se traduzca en algunos años en un aumento de la incidencia de cáncer cervicouterino. Las mujeres adultas, en quienes ya no se puede plantear la prevención primaria de la infección, se pueden beneficiar con nuevas tecnologías de tamizaje en base a la detección del VPH o sus 
productos. De acuerdo a la información obtenida de este artículo, las usuarias de ACO requerirían un seguimiento más estrecho.

Agradecimientos: Al personal del consultorio El Roble y a su directora Francis Ciampi por la buena disponibilidad y colaboración en el trabajo de campo. Dr Peter Snijders por el análisis virológico, Dr Rodrigo Prado, Silvia Franceschi y Dr José Manuel Ojeda coinvestigadores en el estudio de prevalencia del año 2001.

\section{Referencias}

1. Parkin M, Bray F, Ferlay J, Pisani P. Global Cancer Statistics, 2002. CA Cancer J Clin 2005; 55: 74-108.

2. Ministerio de Salud, Chile. Guía Clínica Cáncer Cervicouterino 2. 1st Ed. Santiago: Minsal. Santiago 2005.

3. Ministerio de Salud, Chile. Temas de Salud; Estadísticas; Estadísticas Vitales; Mortalidad; Mortalidad por causas; Serie mortalidad por causas; Tumores malignos; Cáncer cervicouterino. Chile.

4. International Union Against Cancer. Prevención del cáncer estrategias basadas en la evidencia. Una guía de la UICC para América Latina. Ginebra; 2006.

5. World Health Organization. IARC Monographs on the Evaluation of Carcinogenic Risks to Humans. Human papillomaviruses. Summary of Data Reported and Evaluation 1995; 64.

6. World Health Organization. IARC Monographs on the Evaluation of Carcinogenic Risks to Humans.VOLUME 90 Human Papillomaviruses. 2007.

7. Muñoz N, Kato I, Bosh X. Risk Factors for HPV DNA Detection in Middle-Aged Women. Sexually Transmitted Diseases 1996; 23:504-510.

8. Castellsague X, De Sanjose S. Transmission des HPV. Papillomavirus humains. Biologie et pathologie tumorale, Paris, Editons Tec \& Doc, Editions Médicales Internationales 2003: 309-33.

9. Muñoz N, Francechi S, Bosetti C. Role of parity and human papillomavirus in cervical cancer:the IARC multicentric case- control study. The Lancet 2000; 359: 1093-2000.

10. Moreno V, Bosch F, Muñoz N. Effect of oral contraceptives on risk of cervical cancer in women with human papillomavirus infection: the IARC multicentric casecontrol study. Lancet 2002; 359: 1085-92.

11. Anne O, Olsen JD, Anders S, Per M. Combined Effect of Smoking and Human Papillomavirus Type 16 Infection in Cervical Carcinogenesis. Epidemiology 1998; 9: 346-9.

12. Hildesheim A, Herrero R, Castle P. HPV co-factors related to the development of cervical cancer: results from a population-based study in Costa Rica. Br J Cancer 2001; 84: 1219-26.

13. Ferreccio C, Prado R, Luzoro A, Ampuero S, Snijders P. Population based prevalence and age distribution of HPV among women in Santiago, Chile. Cancer Epidem, Biomarkers and Prev 2004; 13.

14. Thrall MJ, Pambuccian SE, Stelow EB, McKeon DM, Miller L, Savik K, Gulbahce HE. Impact of the more restrictive definition of atypical squamous cells introduced by the 2001 Bethesda System on the sensitivity and specificity of the Papanicolaou test: a 5-year followup study of Papanicolaou tests originally interpreted as ASCUS, reclassified according to Bethesda 2001 criteria. Cancer 2008; 114: 171-9.

15. de Roda Husman AM, Walboomers JM, van de Brule AJ, Meijer CJ, Snijders PJ. The use of general primers GP5 and GP6 elongated at their 3' adjacent highly conserved sequences improves human detection by PCR. J Gen Virol 1995; 76: 1057-62.

16. van den Brule AJ, Pol P, Fransen-Daalmeijer N, Schouls LM, Meijer CJ, Snijders PJ. GP5+/6+ PCR followed by Reverse Line Blot analysis enables rapid and highthroughput identification of human papillomavirus genotypes. J Clin Micr 2002; 40: 779-87.

17. Muñoz N, Bosch X, De Sanjose S. Epidemiologic Classification of Human Papillomavirus Types Associated with Cervical Cancer. N Engl J Med 2003; 348: 518-27.

18. CONASIDA. ANRS. Estudio nacional de comportamiento sexual. Síntesis de información seleccionada. Ministerio de Salud. Santiago-Chile 2000.

19. De Villiers E.M. Relationship between steroid hormone contraceptives and HPV, cervical intraepithelial neoplasia and cervical carcinoma. Int J Cancer 2003; 103: 705-8.

20. Arrossi S, Sankaranayanan R, Maxwell D. Incidence and mortality of cervical cancer in Latin America. Salud Publica Mex 2003; 45 supl 3: S306-14.

21. World Health Organization. IARC Handbooks of Cancer Prevention. Cervix Cancer Screening. Vol 10. Lyon; 2005. 